\title{
Predicting Pathological Complete Regression with Haematological Markers During Neoadjuvant Chemoradiotherapy for Locally Advanced Rectal Cancer
}

\author{
JOO HO LEE ${ }^{1,2}$, CHANGHOON SONG ${ }^{1}$, SUNG-BUM KANG ${ }^{3}$, \\ HYE SEUNG LEE ${ }^{4}$, KEUN-WOOK LEE ${ }^{5}$ and JAE-SUNG KIM ${ }^{1}$ \\ ${ }^{1}$ Department of Radiation Oncology, Seoul National University College of Medicine, \\ Seoul National University Bundang Hospital, Seongnam, Republic of Korea; \\ ${ }^{2}$ Department of Radiation Oncology, Seoul National University College of Medicine, \\ Seoul National University Hospital, Seoul, Republic of Korea; \\ ${ }^{3}$ Department of Surgery, Seoul National University College of Medicine, \\ Seoul National University Bundang Hospital, Seongnam, Republic of Korea; \\ ${ }^{4}$ Department of Pathology, Seoul National University College of Medicine, \\ Seoul National University Bundang Hospital, Seongnam, Republic of Korea; \\ ${ }^{5}$ Department of Internal Medicine, Seoul National University College of Medicine, \\ Seoul National University Bundang Hospital, Seongnam, Republic of Korea
}

\begin{abstract}
Background: This study evaluated the efficacy of haematological markers for predicting the pathological complete regression $(p C R)$ during and after neoadjuvant chemoradiotherapy (CRT) in patients with locally advanced rectal cancer (LARC). Patients and Methods: A total of 297 patients with LARC underwent neoadjuvant CRT followed by surgical resection. Complete blood counts (CBCs) were performed before CRT, 3 weeks after the start of CRT (intratherapy), and 4 weeks after CRT. Platelet-to-lymphocyte ratio $(P L R)$ and neutrophil-to-lymphocyte ratio (NLR) were calculated using the serial $C B C$ test. The ratio of change in PLR (cPLR) and NLR (cNLR) was calculated as the increase from the pre-therapy value to intra-therapy or post-therapy value divided by the pre-therapy value. Chi-square and t-test for univariate analysis and multivariate logistic regression were performed to identify significant predictors for $p C R$. Receiver operating characteristic (ROC) analysis was used to compare predictive values. Results: The overall rate of pCR was $15.9 \%$. Pre-therapy high haemoglobin and low
\end{abstract}

Correspondence to: Jae-Sung Kim, Department of Radiation Oncology, Seoul National University College of Medicine, Seoul National University Bundang Hospital, 82 Gumi-ro 173beon-gil, Bundang-gu, Seongnam 13620, Republic of Korea. E-mail: jskim@snubh.org

Key Words: Rectal cancer, platelet/lymphocyte ratio, haematological markers, chemoradiotherapy, complete regression.
NLR; intra-therapy high PLR, high NLR, high $C P L R$, and high cNLR; and post-therapy low white blood cell count $(W B C)$, high haemoglobin, and high cPLR were significantly associated with $p C R$. In multivariate logistic regression, pretherapy high haemoglobin [odds ratio $(O R)=1.500$, $p=0.016]$, high intra-therapy PLR $(O R=1.006, p=0.011)$, high intra-therapy $c P L R(O R=4.948, p<0.001)$, and low posttherapy WBC $(O R=0.639, p=0.003)$ were significant predictors for $p C R$. ROC analysis showed that high intratherapy $C P L R$ was the most accurate predictor of $p C R$ (area under the curve $=0.741)$. Conclusion: Changes of PLR during neoadjuvant CRT for LARC are significant predictors of $p C R$.

Neoadjuvant chemoradiotherapy (CRT) followed by total mesorectal excision (TME) has been widely used in patients with locally advanced rectal cancer (LARC) (1). About 15$20 \%$ of patients achieve pathological complete regression (pCR) after neoadjuvant CRT $(1,2)$. Although TME following neoadjuvant CRT exhibits good local control and is known to cause a local recurrence rate of less than $10 \%$ (2), radical surgery may pose significant risks of perioperative morbidities (3). These findings led to the development of a conservative approach for patients with LARC that showed good response to CRT, such as local excision or a 'watchand-wait' approach deferring surgery (4).

However, identification of complete remission after neoadjuvant CRT is a major challenge with the conservative approach (5). The current method for selection of patients for this approach is based on the analysis of the degree of 
clinical regression using colonoscopy and imaging tools (46). However, clinical complete regression shows insufficient correlation with $\mathrm{pCR}$. The discordance rate in the previous reports of the 'watch-and-wait' approach was 8-15\% of patients $(4,6)$. Deferring surgery for these patients with false clinical complete regression may have a significant risk of tumour regrowth. The prediction and identification of pathological regression after neoadjuvant CRT are mandatory for oncological safety.

The significant discordance rate of clinical regression may be related to the risk of microscopic residual disease undetected during preoperative evaluation. In recent years, haematological markers have been studied as surrogates for tumour burden and tumour-host interaction (7-10). Previous studies have reported haematological markers such as the neutrophil-to-lymphocyte ratio (NLR) and platelet-tolymphocyte ratio (PLR) as prognostic markers of disease recurrence and survival outcomes (7-10). We hypothesise that the evaluation of the haematological markers throughout CRT may predict pCR after CRT.

In the present study, we evaluated the application of various haematological markers for the early prediction of pCR after neoadjuvant CRT.

\section{Patients and Methods}

Patients. The present study was approved by the Institutional Ethical Review Board of the Seoul National University Bundang Hospital (IRB no. B-1807-478-106). The medical records of 331 patients with rectal cancer who underwent neoadjuvant CRT followed by TME from January 2004 to August 2015 were retrospectively reviewed. Inclusion criteria were as follows: (i) Pathologically confirmed primary rectal cancer; (ii) clinical T3/T4 or node-positive cases diagnosed by imaging; (iii) neoadjuvant CRT followed by total mesorectal resection; (iv) serial complete blood count (CBC) examination throughout CRT; (v) immunologically competent before and during CRT, including absolute neutrophil count (ANC) $>1,000 / \mu$, white blood cell count (WBC) between 4,000 and $10,000 \times 10^{3} / \mu \mathrm{l}$; (vi) negative for hepatitis $\mathrm{B}$ virus antigen, anti-hepatitis $\mathrm{C}$ virus, anti-human immunodeficiency virus, and venereal disease research laboratory results before CRT; and (vii) no clinical evidence of acute or chronic infection or other autoimmune disease or malignant disease primarily arising from other organs. Total eligible patients meeting the inclusion criteria were 297.

For all patients, the clinical workup included digital rectal examination, routine laboratory tests such as CBC, liver/renal function tests, serology, carcinoembryonic antigen (CEA) level, and colonoscopic examination, including biopsy, computed tomography (CT) evaluating the chest plus abdomen-pelvis, and rectal magnetic resonance imaging (MRI). Eligible patients were pathologically confirmed as having primary rectal cancer before neoadjuvant CRT.

Following the diagnosis of rectal cancer, all 297 patients received neoadjuvant concurrent CRT for rectal cancer. The radiation dose was $45 \mathrm{~Gy}$, followed by a primary tumour boost of 5.4 Gy over a period of 5.5 weeks. Patients were mainly treated with the combination chemotherapy of intravenous bolus 5 -fluorouracil/leucovorin (400/20 $\mathrm{mg} / \mathrm{m}^{2}$ daily injection for 3 days on weeks 1 and 5) or capecitabine $\left(825 \mathrm{mg} / \mathrm{m}^{2}\right)$ twice daily throughout the radiation period. Surgery was performed 4-8 weeks after the end of CRT. Pathologists evaluated the surgical specimens in order to estimate and grade the pathologic response to CRT. pCR was defined as no residual cancer found in histology. Pathological responses were categorised as per the Dworak system (11).

Data collection. CBC was performed for all patients throughout CRT. Laboratory index values, including neutrophil counts, lymphocyte counts, platelet counts, and CEA, were obtained for each patient before CRT (pre-therapy), 3 weeks after the start day of CRT (intra-therapy), and 4 weeks after CRT (post-therapy). Patients were staged according to the classification system of the American Joint Committee on Cancer (7th edition) (12). NLR and PLR were calculated as the ratio of the neutrophil count and of the platelet count divided by the lymphocyte count, respectively. The ratio of change in PLR (cPLR) and NLR (cNLR) was calculated using the following formula:

ratio of change in PLR=(post-therapy or intra-therapy PLR - pretherapy PLR)/pre-therapy PLR, and ratio of change in NLR=(posttherapy or intra-therapy NLR - pre-therapy NLR)/pre-therapy NLR.

Statistical analysis. Univariate analysis was performed with Student's $t$-test and Chi-square test for blood test variables, histological features, and clinical features to find the significant factors related to $\mathrm{pCR}$. Multivariate analysis for predicting $\mathrm{pCR}$ was conducted with logistic regression analysis. cPLR and cNLR were included separately from the fixed values in the multivariate logistic regression, owing to the possible issue of multiple collinearity. A value of $p<0.050$ was considered statistically significant. To compare the predictive power among the significant variables for $\mathrm{pCR}$, the area under the curve (AUC) value was calculated using the receiver operating characteristics (ROC). The cut-off values were determined at the maximum of Youden's index and the maximum of accuracy (13). An AUC value of $>0.7$ was considered sufficient for accurate prediction. Analyses were performed using SPSS version 18 (SPSS Inc., Chicago, IL, USA).

\section{Results}

Patient characteristics and haematological profiles are detailed in Tables I and II. The overall rate of pCR was 15.9\%. Tumour down-staging after neoadjuvant CRT occurred in $58.8 \%$ of patients. As per Dworak classification of tumour regression grade, 49 (16.6\%), 133 (44.9\%), 67 $(22.6 \%)$, and $47(15.9 \%)$ patients were included in grade 1 , 2,3 , and 4 , respectively.

The univariate analysis for pCR is shown in Table III. In the univariate analysis, high pre-therapy haemoglobin $(\mathrm{Hb})$, low pre-therapy NLR, high intra-therapy PLR, high intratherapy NLR, high intra-therapy cPLR, high intra-therapy cNLR, low post-therapy $\mathrm{WBC}$, high post-therapy $\mathrm{Hb}$, and high post-therapy cPLR were significantly associated with pCR $(p=0.005,0.036,0.001,0.004,<0.001,<0.001,<0.001$, 0.047 , and 0.040 , respectively). However, no statistical difference between patients with $\mathrm{pCR}$ and no $\mathrm{pCR}$ was 
Table I. Patient characteristics.

\begin{tabular}{|c|c|c|}
\hline Factor & Subgroup & Value \\
\hline \multirow[t]{2}{*}{ Gender, $\mathrm{n}(\%)$} & Female & $89(30.1)$ \\
\hline & Male & $207(69.9)$ \\
\hline Age, years & Mean (SD) & $61(52-68)$ \\
\hline \multirow[t]{2}{*}{ ECOG PS, n (\%) } & $<2$ & $266(89.9)$ \\
\hline & $\geq 2$ & $30(10.1)$ \\
\hline \multirow[t]{2}{*}{ Distance from anal verge, $\mathrm{n}(\%)$} & $>5 \mathrm{~cm}$ & $117(39.5)$ \\
\hline & $\leq 5 \mathrm{~cm}$ & $179(60.5)$ \\
\hline \multirow[t]{2}{*}{ CEA, n $(\%)$} & $>5 \mathrm{ng} / \mathrm{ml}$ & $194(65.3)$ \\
\hline & $\leq 5 \mathrm{ng} / \mathrm{ml}$ & $103(34.7)$ \\
\hline \multirow[t]{3}{*}{ cStage, n (\%) } & 1 & $5(1.7)$ \\
\hline & 2 & $39(13.2)$ \\
\hline & 3 & $252(85.1)$ \\
\hline \multirow[t]{4}{*}{$\mathrm{cT}, \mathrm{n}(\%)$} & 1 & $1(0.3)$ \\
\hline & 2 & $18(6.1)$ \\
\hline & 3 & $249(84.1)$ \\
\hline & 4 & $28(9.5)$ \\
\hline \multirow[t]{3}{*}{$\mathrm{cN}, \mathrm{n}(\%)$} & 0 & $44(14.9)$ \\
\hline & 1 & $133(44.9)$ \\
\hline & 2 & $119(40.2)$ \\
\hline \multirow[t]{2}{*}{ Radiation modality, n (\%) } & $2 \mathrm{D}$ & $161(54.4)$ \\
\hline & 3D-conformal & $135(45.6)$ \\
\hline \multirow[t]{2}{*}{ Radiation dose, n (\%) } & $50.4 \mathrm{~Gy}$ & $295(99.7)$ \\
\hline & $55.8 \mathrm{~Gy}$ & $1(0.3)$ \\
\hline \multirow[t]{3}{*}{ Chemotherapy, n (\%) } & $5-\mathrm{FU}$ & $97(32.8)$ \\
\hline & Capecitabine & $193(65.2)$ \\
\hline & Other & $6(2.0)$ \\
\hline \multirow[t]{2}{*}{ Type of surgery, n (\%) } & LAR & $264(89.2)$ \\
\hline & APR & $32(10.8)$ \\
\hline \multirow[t]{2}{*}{ Pathology, n (\%) } & Adenocarcinoma & $288(97.3)$ \\
\hline & Mucinous & $8(2.7)$ \\
\hline \multirow[t]{2}{*}{ Resection margin, n (\%) } & Ro & $277(93.6)$ \\
\hline & $\mathrm{R} 1$ & $19(6.4)$ \\
\hline \multirow[t]{2}{*}{ Down-staging, n (\%) } & Yes & $174(58.8)$ \\
\hline & No & $122(41.2)$ \\
\hline \multirow[t]{4}{*}{ Tumour regression grade, $\mathrm{n}(\%)$} & 1 & 49 (16.6) \\
\hline & 2 & $133(44.9)$ \\
\hline & 3 & $67(22.6)$ \\
\hline & 4 & $47(15.9)$ \\
\hline \multirow[t]{5}{*}{ ypT, n (\%) } & 0 & $47(15.9)$ \\
\hline & 1 & $22(7.4)$ \\
\hline & 2 & $79(26.7)$ \\
\hline & 3 & $145(49.0)$ \\
\hline & 4 & $3(1.0)$ \\
\hline \multirow[t]{3}{*}{ ypN, n (\%) } & 0 & $198(66.9)$ \\
\hline & 1 & $77(26.0)$ \\
\hline & 2 & $21(7.1)$ \\
\hline
\end{tabular}

SD: Standard deviation; CEA: carcinoembryonic antigen; LAR: low anterior resection; APR: abdominoperineal resection; ECOG PS: Eastern Cooperative Oncology Group performance status; ypT: postchemoradiotherapy pathologic $\mathrm{T}$ stage; ypN: post-chemoradiotherapy pathologic N stage; 5-FU: 5-fluorouracil/leucovorin; cT: clinical T stage; $\mathrm{cN}$ : clinical $\mathrm{N}$ stage.

observed for various clinical factors such as age, Eastern Cooperative Oncology Group performance status, sex, distance from anal verge, clinical $\mathrm{T}$ and $\mathrm{N}$ stage, radiation
Table II. Haematological profile of patients.

\begin{tabular}{lccc}
\hline & \multicolumn{3}{c}{ Mean (range) } \\
\cline { 2 - 4 } Variable & $\begin{array}{c}\text { Before } \\
\text { CRT }\end{array}$ & $\begin{array}{c}\text { During } \\
\text { CRT } \\
\text { (3 weeks) }\end{array}$ & $\begin{array}{c}\text { After } \\
\text { CRT } \\
\text { (4 weeks) }\end{array}$ \\
\hline $\mathrm{WBC}, \times 10^{3 / \mu 1}$ & $6.7(9.9-4)$ & $4.7(14-2)$ & $5.3(17-2.2)$ \\
$\mathrm{Hb}, \mathrm{g} / \mathrm{dl}$ & $14(17-8.5)$ & $13(17-3.9)$ & $13(17-9.1)$ \\
Platelet, $\times 10^{3} / \mu \mathrm{l}$ & $269(653-105)$ & $196(542-14)$ & $237(537-90)$ \\
PLR & $141(554-59)$ & $221(810-23)$ & $232(672-68)$ \\
NLR & $2.1(8.6-0.71)$ & $3.5(13-0.76)$ & $3.5(32-0.91)$ \\
\hline
\end{tabular}

PLR, Platelet to lymphocyte ratio; NLR, neutrophil to lymphocyte ratio; WBC, white blood cell count; Hb, haemoglobin; CRT, chemoradiotherapy.

modality, and CEA. In multivariate logistic regression (Table IV), high pre-therapy $\mathrm{Hb}(p=0.016)$, high intra-therapy PLR $(p=0.011)$, high intra-therapy cPLR $(p<0.001)$, and low posttherapy WBC $(p=0.003)$ were independent and significant predictors for $\mathrm{pCR}$.

To evaluate the most effective haematological marker for pCR prediction, we compared ROC curves for four markers that were found to be significant in the multivariate analysis. Intra-therapy cPLR gave the highest AUC among pre-therapy $\mathrm{Hb}$, intra-therapy PLR, intra-therapy cPLR, and post-therapy WBC (AUC: 0.630, 0.642, 0.741, and 0.661, respectively) (Figure 1). The cut-off value at the maximum of Youden's index was 0.61 for intra-therapy cPLR. At a cut-off value of 0.61 , sensitivity, specificity, accuracy, positive predictive value (PPV), and negative predictive value (NPV) of intratherapy cPLR to predict pCR were $78.7 \%, 62.5 \%, 65.0 \%$, $28.2 \%$, and $94.0 \%$, respectively. The maximum accuracy was obtained at a cut-off value of 1.80 ; at 1.80 , sensitivity, specificity, accuracy, PPV, and NPV of intra-therapy cPLR to predict pCR were $21.3 \%, 98.8 \%, 86.5 \%, 76.9 \%$ and $87.0 \%$, respectively.

\section{Discussion}

In the present study, we evaluated the possible role of haematological markers as predictors of pCR. The prognostic association between inflammation and cancer has been well studied in various types of cancer [reviewed in (14)]. Furthermore, previous studies have reported the significant relationship between inflammatory markers such as neutrophil counts, NLR, and PLR and survival and prognosis of patients with LARC (7-10). However, the data for the correlation between tumour regression and inflammatory makers are inconsistent and limited $(10,15,16)$. Most previous studies used only the pre- and post-treatment values, whereas the present study screened the changes in these values as well as 
Table III. Univariate analysis.

\begin{tabular}{|c|c|c|c|}
\hline Factor & $\begin{array}{c}\mathrm{pCR} \\
\mathrm{N}=47\end{array}$ & $\begin{array}{l}\text { No pCR } \\
\mathrm{N}=250\end{array}$ & $p$-Value \\
\hline \multicolumn{4}{|l|}{ Age, n (\%) } \\
\hline$\leq 60$ Years & $25(53.2 \%)$ & $117(46.8 \%)$ & 0.420 \\
\hline$>61$ Years & $22(46.8 \%)$ & $133(53.2 \%)$ & \\
\hline \multicolumn{4}{|l|}{ ECOG PS, n (\%) } \\
\hline$<2$ & $44(93.6 \%)$ & $223(89.2 \%)$ & 0.360 \\
\hline$\geq 2$ & $3(6.4 \%)$ & $27(10.8 \%)$ & \\
\hline \multicolumn{4}{|l|}{ Gender, n (\%) } \\
\hline Female & $14(29.8 \%)$ & $74(29.6 \%)$ & 0.982 \\
\hline Male & $33(70.2 \%)$ & $176(70.4 \%)$ & \\
\hline \multicolumn{4}{|l|}{$\begin{array}{l}\text { Distance from anal } \\
\text { verge, } \mathrm{n}(\%)\end{array}$} \\
\hline$>5 \mathrm{~cm}$ & $21(44.7 \%)$ & $97(38.8 \%)$ & 0.451 \\
\hline$\leq 5 \mathrm{~cm}$ & $26(55.3 \%)$ & $153(61.2 \%)$ & \\
\hline \multicolumn{4}{|l|}{$\mathrm{cT}, \mathrm{n}(\%)$} \\
\hline $\mathrm{T} 1-2$ & $6(12.8 \%)$ & $13(5.2 \%)$ & 0.125 \\
\hline $\mathrm{T} 3$ & $38(80.9 \%)$ & $212(84.8 \%)$ & \\
\hline $\mathrm{T} 4$ & $3(6.4 \%)$ & $25(10.0 \%)$ & \\
\hline \multicolumn{4}{|l|}{$\begin{array}{l}\text { Clinical node } \\
\text { positive, n (\%) }\end{array}$} \\
\hline No & $11(23.4 \%)$ & $33(13.2 \%)$ & 0.071 \\
\hline Yes & $36(76.6 \%)$ & $217(86.8 \%)$ & \\
\hline \multicolumn{4}{|l|}{$\begin{array}{l}\text { Radiation modality, } \\
\mathrm{n}(\%)\end{array}$} \\
\hline $2 \mathrm{D}$ & $24(51.1 \%)$ & $137(54.8 \%)$ & 0.640 \\
\hline 3D-conformal & $23(48.9 \%)$ & $113(45.2 \%)$ & \\
\hline \multicolumn{4}{|l|}{ CEA, n (\%) } \\
\hline$>5 \mathrm{ng} / \mathrm{ml}$ & $33(70.2 \%)$ & $161(64.4 \%)$ & 0.440 \\
\hline$\leq 5 \mathrm{ng} / \mathrm{ml}$ & $14(29.8 \%)$ & $89(35.6 \%)$ & \\
\hline Before CRT & Mean (SD) & Mean (SD) & \\
\hline $\mathrm{WBC}, \times 10^{3} / \mu \mathrm{l}$ & $6.772(1.436)$ & $6.701(1.439)$ & 0.760 \\
\hline $\mathrm{Hb}, \mathrm{g} / \mathrm{dl}$ & $14.157(1.665)$ & 13.403 (1.669) & 0.005 \\
\hline Platelet count, $\times 10^{3} / \mu \mathrm{l}$ & $260.234(63.979)$ & $270.560(76.269)$ & 0.380 \\
\hline PLR & $121.934(35.896)$ & $144.227(61.404)$ & 0.067 \\
\hline NLR & $1.804(0.710)$ & $2.140(1.046)$ & 0.036 \\
\hline \multicolumn{4}{|l|}{3 Weeks in CRT } \\
\hline $\mathrm{WBC}, \times 10^{3} / \mu \mathrm{l}$ & 4.690 (1.677) & $4.742(1.404)$ & 0.820 \\
\hline $\mathrm{Hb}, \mathrm{g} / \mathrm{dl}$ & $13.183(1.543)$ & $12.856(1.581)$ & 0.193 \\
\hline Platelet count, $\times 10^{3} / \mu \mathrm{l}$ & $194.128(50.321)$ & $196.181(64.083)$ & 0.840 \\
\hline PLR & $266.654(128.116)$ & $212.325(101.988)$ & 0.001 \\
\hline NLR & $4.155(2.157)$ & $3.319(1.761)$ & 0.004 \\
\hline Change in PLR & $1.270(1.105)$ & $0.530(0.569)$ & $<0.001$ \\
\hline Change in NLR & $1.527(1.407)$ & $0.742(0.973)$ & $<0.001$ \\
\hline \multicolumn{4}{|l|}{4 Weeks after CRT } \\
\hline $\mathrm{WBC}, \times 10^{3} / \mu \mathrm{l}$ & $4.420(1.153)$ & $5.419(1.917)$ & $<0.001$ \\
\hline $\mathrm{Hb}, \mathrm{g} / \mathrm{dl}$ & $13.506(1.433)$ & $13.052(1.429)$ & 0.047 \\
\hline Platelet count, $\times 10^{3} / \mu \mathrm{l}$ & $221.085(42.480)$ & $240.260(72.836)$ & 0.081 \\
\hline PLR & $224.651(71.373)$ & $233.611(105.639)$ & 0.580 \\
\hline NLR & $2.816(1.156)$ & $3.595(2.676)$ & 0.051 \\
\hline Change in PLR & $0.958(0.736)$ & $0.725(0.705)$ & 0.040 \\
\hline Change in NLR & $0.730(0.839)$ & $0.901(1.524)$ & 0.452 \\
\hline
\end{tabular}

CEA: Carcinoembryonic antigen; cT: clinical T stage; PLR: platelet to lymphocyte ratio; NLR: neutrophil to lymphocyte ratio; WBC: white blood cell count; Hb: haemoglobin; CRT: chemoradiotherapy; ECOG PS: Eastern Cooperative Oncology Group performance status; pCR: pathological complete regression. Change in PLR or NLR were calculated using the following formula: (post- or intra-therapy value pre-therapy value)/pre-therapy value.
Table IV. Multivariate logistic regression for pathological complete regression.

\begin{tabular}{llc}
\hline Factor & Odds ratio* $(95 \%$ CI $)$ & $p$-Value \\
\hline Hb before CRT, g/dl & $1.500(1.080-2.084)$ & 0.016 \\
NLR before CRT & $0.636(0.387-1.045)$ & 0.074 \\
PLR during CRT & $1.006(1.001-1.010)$ & 0.011 \\
NLR during CRT & $1.188(0.928-1.523)$ & 0.172 \\
WBC after CRT, $\times 10^{3} / \mu 1$ & $0.639(0.474-0.862)$ & 0.003 \\
Hb after CRT, g/dl & $1.081(0.759-1.540)$ & 0.667 \\
Change of PLR during CRT & $4.948(2.074-11.810)$ & $<0.001$ \\
Change of NLR during CRT & $0.923(0.579-1.471)$ & 0.736 \\
Change of PLR after CRT & $0.707(0.395-1.264)$ & 0.242 \\
\hline
\end{tabular}

PLR, Platelet to lymphocyte ratio; NLR, neutrophil to lymphocyte ratio; WBC, white blood cell count; $\mathrm{Hb}$, haemoglobin; CRT, chemoradiotherapy; CI, confidence interval. *Odds ratio represents the incremental odds of pathological complete regression prediction for every unit increase of the factors. Changes of PLR or NLR were calculated using the following formula: (post- or intra-therapy value pre-therapy value)/pre-therapy value.

the intra-therapy values. The degree of tumour regression represents the response to treatment and host-tumour reaction during treatment $(7,17)$. Thus, it is a plausible hypothesis that the change in these values may be a suitable surrogate for the response and change in the disease during CRT. Therefore, detailed analysis with serial evaluation is desirable in order to assess the clinical value of inflammatory markers.

In the present study, the degree of increase in intra-therapy PLR was the most accurate predictor of $\mathrm{pCR}$ among the haematological variables throughout CRT. Lee et al. reported the statistical correlation between pCR and post-therapy cPLR after CRT (10). However, in comparison with the previous study, the present study used multivariate analysis and ROC comparison for various haematological variables to determine the most accurate predictor. Moreover, we excluded patients with possibly confounding immunological factors, including immunodeficiency, and those that missed blood testing at the pre-determined time points. As a result, intra-therapy cPLR, newly defined in the present study, was superior to posttherapy cPLR and allowed for the early prediction of response during CRT. In addition to the prediction of $\mathrm{pCR}$ with the conservative approach, response assessment during CRT may provide a chance for modification of the treatment strategy or escalation of CRT intensity for non-responding patients (18). To our knowledge, this is the first study to evaluate the predictive value of haematological changes during CRT in immunocompetent patients with LARC.

Positron-emission tomography with CT (PET-CT) and MRI are the most actively studied approaches for pCR prediction (19). Prediction with PET-CT and MRI before CRT showed inconsistent correlation with pCR (19-21). PET-CT evaluation 

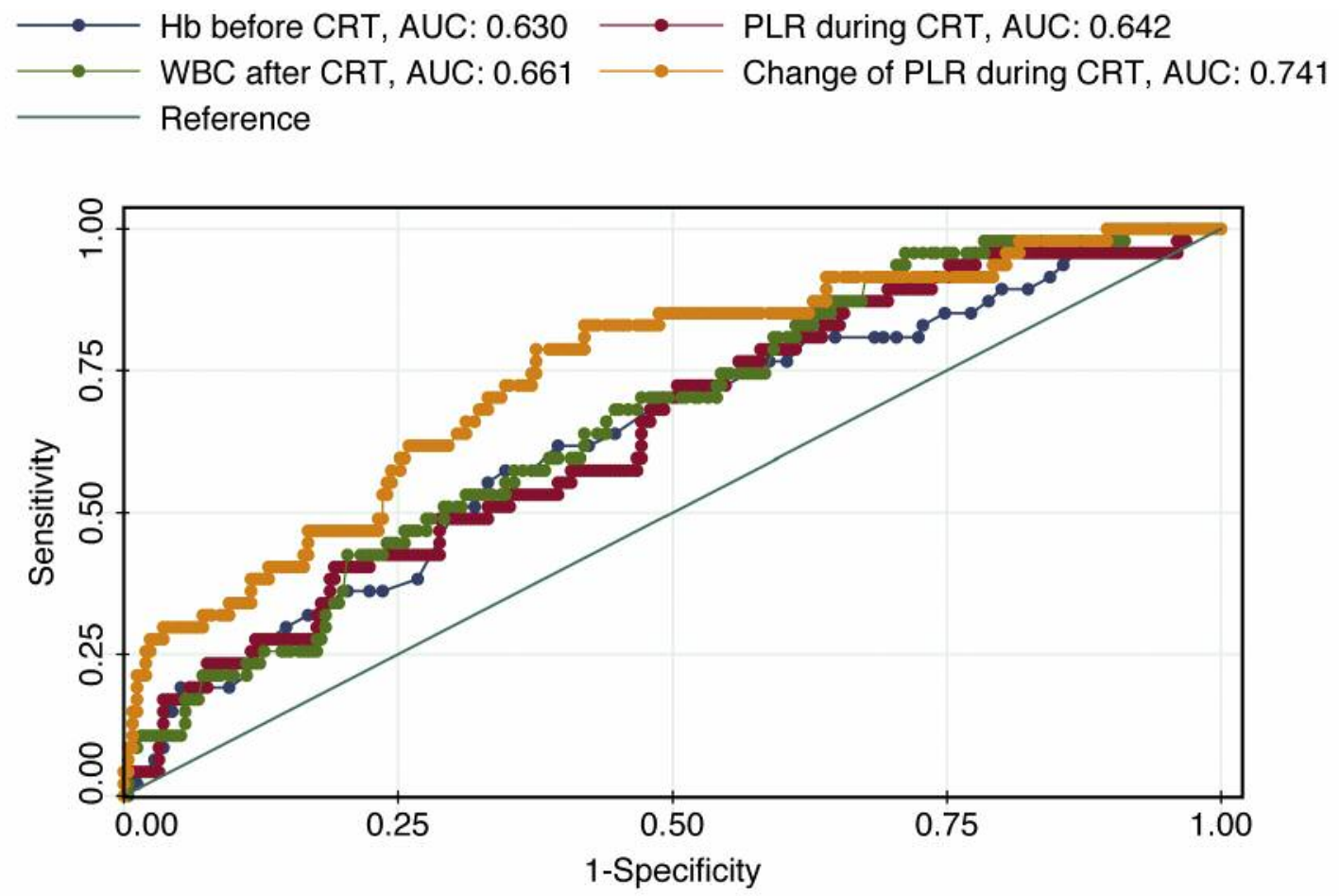

Figure 1. Receiver operating characteristic analysis comparing the ability of haematological markers to predict pathological complete regression. The area under the curve (AUC) is shown in the legend. Hb, Haemoglobin; WBC, white blood cell; PLR, platelet to lymphocyte ratio.

during CRT seems more promising, as evident from an accuracy of $82-95 \%(22,23)$. However, the high cost and effort of serial imaging with PET-CT throughout CRT would limit the sample size necessary to define the protocol and cut-off value as well as clinical availability. In the present study, the large sample size of immunocompetent patients showed comparable predictive power and resulted in the high accuracy of ROC analysis (86.5\%) and high NPV (94.0\%) at maximum Youden's index. The major concern raised by conservative approaches, such as omitting surgery is under-treating patients with false complete regression $(1,5)$. Clinical availability and high NPV of intratherapy cPLR may be a valuable option to ensure oncological safety during the deintensification of treatment.

However, several limitations need to be addressed while using inflammatory markers for the prediction of pCR in clinics. Firstly, a detailed protocol should be established to unify sampling time points and methods. Although the present study used the tri-sampling method (pre-therapy, intra-therapy, and post-therapy), the most sensitive sampling during CRT to predict pCR could not be specified. Secondly, ensuring the quality of clinical laboratory practice is important to ensure consistent results among the studies (24). Thirdly, more direct evaluation of the host-tumour response may be obtained with circulating tumour cell or DNA, tumour-infiltrating lymphocytes, and molecular profiling of peripheral lymphocytes $(25,26)$, although the clinical availability of the CBC test is superior. Taken together, a prospective study overcoming these limitations is desirable in order to establish the standard of application using haematological markers.

In conclusion, the present study suggests that the change in PLR during neoadjuvant CRT may be used as a predictive marker for pCR in patients with LARC.

\section{Acknowledgements}

This research was supported by Basic Science Research Program through the National Research Foundation of Korea (NRF) funded by the Ministry of Education (Grant number: NRF-2017R1D1A1B 03033892).

\section{References}

1 Kim JH: Controversial issues in radiotherapy for rectal cancer: A systematic review. Radiat Oncol J 35: 295-305, 2017.

2 Sauer R, Liersch T, Merkel S, Fietkau R, Hohenberger W, Hess C, Becker H, Raab HR, Villanueva MT, Witzigmann H, Wittekind C, Beissbarth T and Rodel C: Preoperative versus postoperative chemoradiotherapy for locally advanced rectal cancer: results of the German $\mathrm{CAO} / \mathrm{ARO} / \mathrm{AIO}-94$ randomized phase III trial after a median follow-up of 11 years. J Clin Oncol 30: 1926-1933, 2012. 
3 Paun BC, Cassie S, MacLean AR, Dixon E and Buie WD: Postoperative complications following surgery for rectal cancer. Ann Surg 251: 807-818, 2010.

4 Habr-Gama A, Perez RO, Nadalin W, Sabbaga J, Ribeiro U Jr., Silva e Sousa AH, Jr., Campos FG, Kiss DR and GamaRodrigues J: Operative versus nonoperative treatment for stage 0 distal rectal cancer following chemoradiation therapy: Longterm results. Ann Surg 240: 711-718, 2004.

5 Bernier L, Balyasnikova S, Tait D and Brown G: Watch-andwait as a therapeutic strategy in rectal cancer. Curr Colorectal Cancer Rep 14: 37-55, 2018.

6 Creavin B, Ryan E, Martin ST, Hanly A, O'Connell PR, Sheahan $\mathrm{K}$ and Winter DC: Organ preservation with local excision or active surveillance following chemoradiotherapy for rectal cancer. Br J Cancer 116: 169-174, 2017.

7 Carruthers R, Tho LM, Brown J, Kakumanu S, McCartney E and McDonald AC: Systemic inflammatory response is a predictor of outcome in patients undergoing preoperative chemoradiation for locally advanced rectal cancer. Colorectal Dis 14: e701-e707, 2012.

8 Yang J, Xu H, Guo X, Zhang J, Ye X, Yang Y and Ma X: Pretreatment inflammatory indexes as prognostic predictors for survival in colorectal cancer patients receiving neoadjuvant chemoradiotherapy. Sci Rep 8: 3044, 2018.

9 Bozkaya Y, Özdemir NY, Erdem GU, Güner EK, Ürün Y, Demirci NS, Yazıcı O, Köstek $\mathrm{O}$ and Zengin N: Clinical predictive factors associated with pathologic complete response in locally advanced rectal cancer. J Oncol Sci 4: 5-10, 2018.

10 Lee IH, Hwang S, Lee SJ, Kang BW, Baek D, Kim HJ, Park SY, Park JS, Choi GS, Kim JC, Cho SH and Kim JG: Systemic inflammatory response after preoperative chemoradiotherapy can affect oncologic outcomes in locally advanced rectal cancer. Anticancer Res 37: 1459-1465, 2017.

11 Song C, Chung JH, Kang SB, Kim DW, Oh HK, Lee HS, Kim JW, Lee KW, Kim JH and Kim JS: Impact of tumor regression grade as a major prognostic factor in locally advanced rectal cancer after neoadjuvant chemoradiotherapy: A proposal for a modified staging system. Cancers (Basel) 10: pii: E319, 2018.

12 Edge SB, Byrd DR, Compton CC, Fritz AG, Greene FL and Trotti A: AJCC Cancer Staging Manual.Seventh Edition. New York: Springer-Verlag; 2010.

13 Bohning D, Holling $\mathrm{H}$ and Patilea V: A limitation of the diagnostic-odds ratio in determining an optimal cut-off value for a continuous diagnostic test. Stat Methods Med Res 20: 541-550, 2011

14 McMillan DC: Systemic inflammation, nutritional status and survival in patients with cancer. Curr Opin Clin Nutr Metab Care 12: 223-226, 2009.

15 Shen L, Zhang H, Liang L, Li G, Fan M, Wu Y, Zhu J and Zhang Z: Baseline neutrophil-lymphocyte ratio $(>/=2.8)$ as a prognostic factor for patients with locally advanced rectal cancer undergoing neoadjuvant chemoradiation. Radiat Oncol 9: 295, 2014.

16 Krauthamer M, Rouvinov K, Ariad S, Man S, Walfish S, Pinsk I, Sztarker I, Charkovsky T and Lavrenkov K: A study of inflammation-based predictors of tumor response to neoadjuvant chemoradiotherapy for locally advanced rectal cancer. Oncology 85: 27-32, 2013.
17 Demaria S and Formenti SC: Role of T-lymphocytes in tumor response to radiotherapy. Front Oncol 2: 95, 2012.

18 Lee JH, Chie EK, Jeong SY, Kim TY, Kim DY, Kim TH, Kim SY, Baek JY, Chang HJ, Kim MJ, Park SC, Oh JH, Kim SH, Lee JH, Choi DH, Park HC, Kang SB and Kim JS: Redefining the positive circumferential resection margin by incorporating preoperative chemoradiotherapy treatment response in locally advanced rectal cancer: A multicenter validation study. Cancer Res Treat 50: 506-517, 2018.

19 Joye I, Deroose CM, Vandecaveye V and Haustermans K: The role of diffusion-weighted MRI and (18)F-FDG PET/CT in the prediction of pathologic complete response after radiochemotherapy for rectal cancer: A systematic review. Radiother Oncol 113: 158165,2014

20 Lambregts DM, Rao SX, Sassen S, Martens MH, Heijnen LA, Buijsen J, Sosef M, Beets GL, Vliegen RA and Beets-Tan RG: MRI and Diffusion-weighted MRI volumetry for identification of complete tumor responders after preoperative chemoradiotherapy in patients with rectal cancer: A bi-institutional validation study. Ann Surg 262: 1034-1039, 2015.

21 Guillem JG, Ruby JA, Leibold T, Akhurst TJ, Yeung HW, Gollub MJ, Ginsberg MS, Shia J, Suriawinata AA, Riedel ER, Mazumdar M, Saltz LB, Minsky BD, Nash GM, Paty PB, Temple LK, Weiser MR and Larson SM: Neither FDG-PET nor CT can distinguish between a pathological complete response and an incomplete response after neoadjuvant chemoradiation in locally advanced rectal cancer: a prospective study. Ann Surg 258: 289-295, 2013.

22 Lambrecht M, Deroose C, Roels S, Vandecaveye V, Penninckx F, Sagaert X, van Cutsem E, de Keyzer F and Haustermans K: The use of FDG-PET/CT and diffusion-weighted magnetic resonance imaging for response prediction before, during and after preoperative chemoradiotherapy for rectal cancer. Acta Oncol 49: 956-963, 2010

23 Goldberg N, Kundel Y, Purim O, Bernstine H, Gordon N, Morgenstern S, Idelevich E, Wasserberg N, Sulkes A, Groshar $\mathrm{D}$ and Brenner B: Early prediction of histopathological response of rectal tumors after one week of preoperative radiochemotherapy using 18 F-FDG PET-CT imaging. A prospective clinical study. Radiat Oncol 7: 124, 2012.

24 Carraro P and Plebani M: Errors in a stat laboratory: types and frequencies 10 years later. Clin Chem 53: 1338-1342, 2007.

25 Schou JV, Larsen FO, Sorensen BS, Abrantes R, Boysen AK, Johansen JS, Jensen BV, Nielsen DL and Spindler KL: Circulating cell-free DNA as predictor of treatment failure after neoadjuvant chemo-radiotherapy before surgery in patients with locally advanced rectal cancer. Ann Oncol 29: 610-615, 2018.

26 Teng F, Mu D, Meng X, Kong L, Zhu H, Liu S, Zhang J and Yu $\mathrm{J}$ : Tumor infiltrating lymphocytes (TILs) before and after neoadjuvant chemoradiotherapy and its clinical utility for rectal cancer. Am J Cancer Res 5: 2064-2074, 2015.

Received October 16, 2018

Revised October 24, 2018

Accepted October 26, 2018 